\title{
Osteochondral Regeneration of the Loading-bearing Site Using a Scaffold Free Three-dimensional Construct of Adipose Tissue-derived Mesenchymal Stem Cells in Pigs
}

Daiki Murata' ${ }^{1}$, Satoshi Tokunaga ${ }^{1}$, Shizuka Akieda ${ }^{2}$, Koichi Nakayama ${ }^{3}$, Kentaro Setoyama ${ }^{4}$, Makoto Fujiki ${ }^{1}$ and Kazuhiro Misumi ${ }^{1 *}$

${ }^{1}$ Kagoshima University, Joint Faculty of Veterinary Medicine, Department of Veterinary Clinical Science, Veterianry Surgery, Kagoshima, Japan

${ }^{2}$ Cyfuse Biomedical K.K, Fukuoka, Japan

${ }^{3}$ Saga University, Faculty of Medicine, Department of Regenerative Medicine and Biomedical Engineering, Saga, Japan

${ }^{4}$ Kagoshima University, Frontier Science Research Center, Kagoshima, Japan

\begin{abstract}
Background: Many surgical strategies for reconstruction of both bone and cartilage have ever been investigated to restore joint structure and function in the late stages of Osteoarthritis $(\mathrm{OA})$. This study was designed to investigate the regeneration of articular cartilage and subchondral bone in the loading-bearing site using a three-dimensional (3D) construct of autologous adipose tissue-derived mesenchymal stem cells (AT-MSCs).
\end{abstract}

Methods: A 3D construct consisting of approximately 1,920 spheroids each containing $5.0 \times 10^{4}$ AT-MSCs was implanted into an osteochondral defect (with a diameter of $6.8 \mathrm{~mm}$ and a depth of $6 \mathrm{~mm}$ ) in the right femoral medial condyle in five adult mini-pigs. The contralateral (left femoral) defect was the control. At three and six months post-operatively, the defects were evaluated using both CT and MR imaging. The radiolucent volume (RV, $\left.\mathrm{mm}^{3}\right)$ of the defects was calculated based on the multiplanar reconstruction of the CT images. MR images and gross and histologic pathology features were scored using a modified-MOCART system and the ICRS system, respectively, at six months post-operatively.

Results: The percentages of RVs at three and six months compared with those immediately after the surgeries were significantly decreased in the implanted defects compared with the control defects. The total scores of modifiedMOCART system were also significantly increased in the implanted sites comparing to the controls. Although there were no statistical differences in the average of gross scores, the average histological scores were significantly higher in the implanted sites than in the control sites.

Conclusion: This is the first report suggesting that implantation of a scaffold-free three dimensional construct of only AT-MSCs into the osteochondral defect regenerates the original cartilage and subchondral bone structures over six months post-operatively in the loading-bearing site of large animal.

Keywords: Adipose tissue; Bone; Cartilage; Scaffold free; Stem cells; Swine; Three-dimensional; Regeneration

\section{Introduction}

Osteoarthritis (OA), defined as cartilage degradation and subchondral bone sclerosis/deformity, is slowly exacerbated not only following cumulative injuries to bone, cartilage, ligament, and the other joint structures [1], but also due to many factors related to senescence, body mass index, and life-style [2]. To restore joint structure and function in the late stages of $\mathrm{OA}$, surgical strategies for reconstruction of both bone and cartilage have been investigated. A clinical report on osteochondral autografts (mosaicplasty) from non-load-bearing sites into the deteriorated sites (loading-bearing sites) showed favorable outcomes following surgery (clinical improvement in $79-94 \%$ of OA patients) [3]. However, with this therapeutic strategy, the loss of clinically sound cartilage is inevitable, and the obtainable autografts are limited by the large size of the osteochondral defects. Alternatively, a combination of artificial bone and autologous chondrocytes seeded onto a collagen scaffold has also shown favorable restoration of bone and cartilage [4]. New bone can be produced following the implantation of bone filler materials (for example, beta-tricalcium phosphate and/or hydroxyapatite) combined with or without bone-inducing substances, because crucial players in bone, the osteoclasts and osteoblasts, degrade the materials and generate the original bone matrix. On the other hand, articular cartilage has been shown to have a lower self-restorative capacity due to its avascularity and hypocellularity that could also be inappropriate for degrading the artificial materials such as the cell- scaffolds. The materials that could persist in implanted sites for long periods are unsuitable for regenerating articular cartilage consisting of the pure hyaline cartilage, and therefore, we hypothesize that scaffoldfree cell implants are more effective. Previous studies on autologous chondrocyte implantation have revealed problems such as isolation of a few chondrocytes from large amount of normal cartilage [5], the small number of obtainable chondrocytes after in vitro culture [5] and dedifferentiation of chondrocytes during the culture passage [6]. To resolve these problems, studies on bone and cartilage regeneration using stem cells have recently been reported by Tatebe et al. [7]. Mesenchymal stem cells (MSCs) derived from bone marrow (BM), adipose tissue (AT), and synovium (SM) have been shown to differentiate into bone

${ }^{*}$ Corresponding author: Kazuhiro Misumi, Veterinary Surgery, Department of Veterinary Clinical Science, Faculty of Veterinary Medicine, Kagoshima University, 21-24 Korimoto 1-chome, Kagoshima 890-0065, Japan, Tel: +81-(0)99-285-8730 Fax: +81-(0)99-285-8730; E-mail: kaz_msm@vet.kagoshima-u.ac.jp

Received February 15, 2016; Accepted February 23, 2016; Published March 03 2016

Citation: Murata D, Tokunaga S, Akieda S, Nakayama K, Setoyama K, et al. (2016) Osteochondral Regeneration of the Loading-bearing Site Using a Scaffold Free Three-dimensional Construct of Adipose Tissue-derived Mesenchymal Stem Cells in Pigs. J Tissue Sci Eng 7: 165. doi:10.4172/2157-7552.1000165

Copyright: ( 2016 Murata D, et al. This is an open-access article distributed under the terms of the Creative Commons Attribution License, which permits unrestricted use, distribution, and reproduction in any medium, provided the original author and source are credited. 
Citation: Murata D, Tokunaga S, Akieda S, Nakayama K, Setoyama K, et al. (2016) Osteochondral Regeneration of the Loading-bearing Site Using a Scaffold Free Three-dimensional Construct of Adipose Tissue-derived Mesenchymal Stem Cells in Pigs. J Tissue Sci Eng 7: 165. doi:10.4172/2157-7552.1000165

and cartilage in vitro [8-10]. Among them, we expect that a therapeutic strategy using AT-MSCs would be acceptable for many OA patients. AT plays crucial roles in energy storage, shock absorbance, heat insulation, and endocrine function, but it might be often too much for OA patients with obesity and increased body mass index. If the AT that is eliminated to reduce a patient's weight can be utilised as a source of autologous MSCs, the regenerative strategy of bone and cartilage involving the use of AT-MSCs could be the most reasonable application for OA patients. Although intrapatellar fat pad also might be an useful cell sauce of AT-MSCs, it could not be acceptable to incise the knee joint for obtaining AT. It has been reported that AT-MSCs could not differentiate into chondrocytes as well as SM-MSCs [11]. However, a recent study using rabbits and pigs presented bone and cartilage regeneration after implanting a scaffold-free three-dimensional (3D) construct of AT-MSCs into an osteochondral defect [12,13]. These studies were designed to evaluate the autologous implantation in nonloading-bearing sites, where the osteochondral defects were generated subsequent to the mosaicplasty. Therefore, the aim of this study was to evaluate the regeneration of articular cartilage and subchondral bone after implanting a 3D construct of autologous AT-MSCs in the loadingbearing site, where the osteocartilage may be mainly deteriorated in OA.

\section{Materials and Method}

\section{Animals}

Five mini-pigs (Nippon Institute for Biological Science), denoted animal Nos. 1 to 5, were used in this study. Their body weights and ages were $25.6 \mathrm{~kg}$ and 22 months (No. 1), $25.4 \mathrm{~kg}$ and 22 months (No. 2), $27.6 \mathrm{~kg}$ and 22 months (No. 3), $25.6 \mathrm{~kg}$ and 22 months (No. 4) and 26.0 $\mathrm{kg}$ and 22 months (No. 5). All procedures in this study were approved by the Animal Care and Use Committee of Kagoshima University (Approval No. D13025).

\section{Isolation and expansion of AT-MSCs}

Fifteen to twenty grams of cervical adipose tissue per animal was aseptically obtained under general anesthesia. The adipose tissues were minced and digested for $90 \mathrm{~min}$ in phosphate-buffered saline (PBS) containing $0.1 \%$ collagenase type I (Worthington Biochemical). The digested cell suspensions were filtered through a $70 \mu \mathrm{m}$ pore diameter membrane (BD) and centrifuged at $160 \times \mathrm{g}$ for $5 \mathrm{~min}$ at room temperature. After decanting the supernatant, the pellet was rinsed with PBS and centrifuged. After removing the supernatant, the pellet was resuspended and plated in a $150 \mathrm{~cm}^{2}$ culture dish (TPP) in complete culture medium (CCM): Dulbecco's modified Eagle medium (DMEM, Life Technologies) containing 10\% fetal bovine serum (FBS, Thermo Fisher Scientific) and $1 \%$ antibiotic-mycotic (Life Technologies). Following incubation at $37{ }^{\circ} \mathrm{C}$ in $5 \% \mathrm{CO}_{2}$ for seven days, the cells adhering to the bottom of the dish were washed with PBS and cultured in CCM. The medium was changed on the $7^{\text {th }}$ day (D7) in Passage 0 (P0). The cells were harvested with $0.25 \%$ trypsin and $1 \mathrm{mM}$ ethylenediaminetetraacetate (EDTA, Life technologies), diluted by adding five volumes of PBS, and centrifuged on the $10^{\text {th }}$ day (D10). After decanting the supernatant, the pellet was rinsed with CCM, and the cells were replated at $5 \times 10^{5}$ cells in $150 \mathrm{~cm}^{2}$ dishes and cultured for six days. The medium was changed every three days over six days in P1. This serial process of passaging was repeated until the analysis and the creation of a plug.

\section{Preparation of 3D construct of AT-MSCs}

The general outline of this method of construction has been previously reported [12-14]. At least $1 \times 10^{8}$ AT-MSCs were used to produce an autologous construct. The cells were inoculated into twenty 96-well plates (Sumitomo bakelite) with $5 \times 10^{4}$ cells per well. After the plates were incubated undisrupted for $48 \mathrm{~h}$, the cells formed a spheroid with a diameter of approximately $700 \mu \mathrm{m}$ in the bottom of the well. Approximately 1,920 spheroids were placed into a cylindrical mold and incubated in CCM until implantation (7 days). When the mold was carefully removed, a columnar construct that was $6.3 \mathrm{~mm}$ in diameter and $6 \mathrm{~mm}$ in height appeared and was used for the subsequent autologous implantation (Figure 1a).

\section{Implantation of the 3D construct of AT-MSCs}

The implant surgery was performed under general anesthesia using oxygen and isoflurane inhalation following pre-medication with sedatives (medetomidine $50 \mathrm{\mu g} /$ $\mathrm{kg}$ intramuscular injection and midazoram $250 \mu \mathrm{g} / \mathrm{kg}$ intramuscular injection) and analgesics (butorphanol $100 \mu \mathrm{g} / \mathrm{kg}$ intramuscular injection and ketamine $5 \mathrm{mg} / \mathrm{kg}$ intramuscular injection). Both femorotibial joints were incised from the outside, and the femoral medial condyle was exteriorized. Using a surgical trephine with an outer diameter of $6.8 \mathrm{~mm}$, the articular cartilage and the subchondral bone were harvested to a depth of $6 \mathrm{~mm}$ in the center of the medial chondyle. By removing a column of cartilage and bone, a cylindrical osteochondral defect was created in each medial condyle (Figure 1b). A columnar construct ( $6.3 \mathrm{~mm}$ in diameter and $6 \mathrm{~mm}$ in height) composed of spheroids of AT-MSCs was autografted into the osteochondral defect in the right hind limb (Figure 1c), and nothing was implanted into the left limbs (control defects, Figure 1b). The gap between the defect (diameter of $6.8 \mathrm{~mm}$ ) and the construct (that of $6.3 \mathrm{~mm}$ ) was filled up by a press fit after implantation.

\section{CT assessment of the osteochondral defects}

The implants and the osteochondral defects were followed up every three months for six months (zero, three, and six months) after surgery using a computed tomography (CT) scanner (TOSHIBA) in both stifles. The animals were sedated by medetomidine and midazoram as descrived above, and then retained at the dorsal position. Their both stifles were examined by taking a series of x-rays (a CT scan) and analyzed using a MRP images to reconstruct 2-D and 3-D images of the interior surfaces of the bone defects. As shown in the figures, longitudinal section images were obtained in the lateral views of the cylindrical defect with $0.5 \mathrm{~mm}$ slice thickness. The radiolucent area of
A Columnar construct
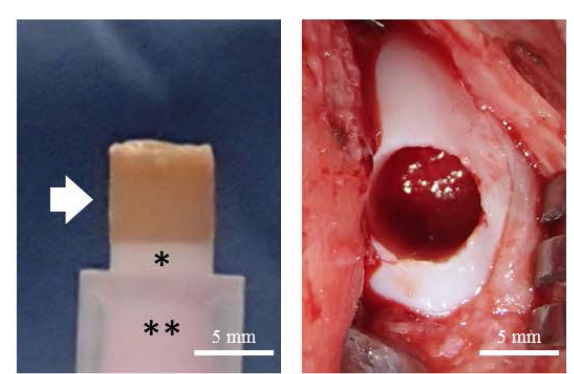

C Implanted defect

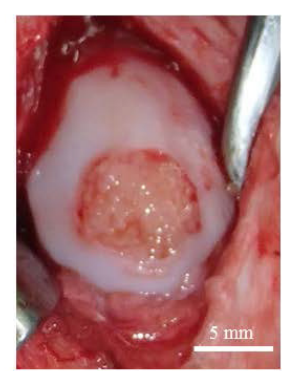

Figure 1: Surgical procedure. Columnar construct $(6.3 \mathrm{~mm}$ in diameter and 6 $\mathrm{mm}$ in height) for the implantation

(a). A cylindrical osteochondral defect in each medial condyle before implantation (b). The construct composed of approximately 1,920 spheroids of AT-MSCs was autografted into the osteochondral defect in the right hind limb (c). Nothing was implanted into the left limbs (control defects; B). The white portions under the construct are a construct sill (asterisk) and a mold (double asterisks). 
Citation: Murata D, Tokunaga S, Akieda S, Nakayama K, Setoyama K, et al. (2016) Osteochondral Regeneration of the Loading-bearing Site Using a Scaffold Free Three-dimensional Construct of Adipose Tissue-derived Mesenchymal Stem Cells in Pigs. J Tissue Sci Eng 7: 165. doi:10.4172/2157-7552.1000165

Page 3 of 8

the defect in each 2-D sectional image was measured, and these areas were integrally calculated to a volume of the defect.

\section{MR assessment of the osteochondral defects}

The implants and the osteochondral defects were followed up every three months for six months (zero, three, and six months) after surgery using a magnetic resonance (MR) 3.0 tesla imager (HITACH) in both stifles. The animals were sedated by medetomidine and midazoram as descrived above, and then retained at the dorsal position to be set up in the coil for human head. The findings of MR images were also scored using a modified 2D-MOCART grading scale (Table 1) [15].

\section{Pathological assessment of the osteochondral defects}

All mini-pigs were euthanized six months after surgery, and the macroscopic findings were scored using the ICRS gross grading scale (Table 2). Both distal femurs were fixed in $10 \%$ NBF for two weeks and then longitudinally sectioned parallel to the trochlear groove. The tissue was paraffin embedded following decalcification with formic acid for one week. Serial sections $(6-\mu \mathrm{m}$ thick) were placed on glass slides and evaluated with hematoxylin \& eosin (HE) staining, Masson's trichrome staining, safranin $\mathrm{O}$ staining, and immunohistochemistry using specific antibodies against collagen type II (1:100 dilution, Daiichi Fine Chemicals) and an Avidin-Biotin Enzyme Complex system (VECTASTAIN ABC Standard Kit, Vector Laboratories). The histopathological findings were also scored using the ICRS histological grading scale (Table 3 ).

\section{Statistical analysis}

All numeric data are presented as the mean \pm standard deviation

\begin{tabular}{|c|c|c|}
\hline \multicolumn{2}{|r|}{ Feature } & \multirow{2}{*}{$\begin{array}{c}\text { Score } \\
20\end{array}$} \\
\hline \multirow{5}{*}{ Defect fill } & Complete & \\
\hline & Hypertrophy & 15 \\
\hline & Incomplete $>50 \%$ & 10 \\
\hline & Incomplete $<50 \%$ & 5 \\
\hline & Subchondral bone expose & 0 \\
\hline \multirow{4}{*}{ Cartilage interface } & Complete & 15 \\
\hline & Demarcating border visible & 10 \\
\hline & Defect visible $<50 \%$ & 5 \\
\hline & Defect visible $>50 \%$ & 0 \\
\hline \multirow{3}{*}{ Surface } & Surface intact & 10 \\
\hline & Surface damaged $<50 \%$ of depth & 5 \\
\hline & Surface damaged $>50 \%$ of depth & 0 \\
\hline \multirow{2}{*}{ Adhesion } & Yes & 5 \\
\hline & No & 0 \\
\hline \multirow{2}{*}{ Structure } & Homogenous & 5 \\
\hline & Inhomogenous or cleft formation & 0 \\
\hline \multirow{3}{*}{ Signal intensity } & Normal & 30 \\
\hline & Nearly normal & 10 \\
\hline & Abnormal & 0 \\
\hline \multirow{2}{*}{ Subchondral Lamina } & Intact & 5 \\
\hline & Not intact & 0 \\
\hline \multirow{2}{*}{ Subchondral bone } & Intact & 5 \\
\hline & Granulation tissue, cyst, sclerosis & 0 \\
\hline \multirow{2}{*}{ Effusion } & No effusion & 5 \\
\hline & Effusion & 0 \\
\hline \multicolumn{2}{|l|}{ Total } & $(0-100)$ \\
\hline
\end{tabular}

Tabel 1: Modified 2D-MOCART scores.

\begin{tabular}{|c|c|c|}
\hline \multicolumn{2}{|c|}{ Feature } & \multirow{2}{*}{$\begin{array}{c}\text { Score } \\
4\end{array}$} \\
\hline \multirow{5}{*}{ Coverage } & $>75 \%$ fill & \\
\hline & $50-75 \%$ fill & 3 \\
\hline & $25-50 \%$ fill & 2 \\
\hline & $<25 \%$ fill & 1 \\
\hline & No fill & 0 \\
\hline \multirow{5}{*}{ Neocartilage color } & Normal & 4 \\
\hline & $25 \%$ yellow/brown & 3 \\
\hline & $50 \%$ yellow/brown & 2 \\
\hline & $75 \%$ yellow/brown & 1 \\
\hline & $100 \%$ yellow/brown & 0 \\
\hline \multirow{5}{*}{ Defect margins } & Invisible & 4 \\
\hline & $25 \%$ circumference visible & 3 \\
\hline & $50 \%$ circumference visible & 2 \\
\hline & $75 \%$ circumference visible & 1 \\
\hline & Entire circumference visible & 0 \\
\hline \multirow{5}{*}{ Surface } & Smooth/level with normal & 4 \\
\hline & Smooth but raised & 3 \\
\hline & Irregular $25-50 \%$ & 2 \\
\hline & Irregular 50-75\% & 1 \\
\hline & Irregular $>75 \%$ & 0 \\
\hline \multicolumn{2}{|l|}{ Average } & $(0-4)$ \\
\hline
\end{tabular}

Tabel 2: ICRS gross grading scale.

\begin{tabular}{|c|c|c|}
\hline \multicolumn{2}{|r|}{ Feature } & \multirow{2}{*}{$\begin{array}{c}\text { Score } \\
3\end{array}$} \\
\hline & Smooth/continuous & \\
\hline surlace & Discontinuities/irregulatrity & 0 \\
\hline \multirow{4}{*}{ Matrix } & Hyaline & 3 \\
\hline & Mixture; hyaline/fibrocartilage & 2 \\
\hline & Fibrocartilage & 1 \\
\hline & Fibrous tissue & 0 \\
\hline \multirow{4}{*}{ Cell distribution } & Columnar & 3 \\
\hline & Mixed/columnar clusters & 2 \\
\hline & Clusters & 1 \\
\hline & Individual cells/disorganized & 0 \\
\hline \multirow{3}{*}{ Viability of cell population } & Predominantly viable & 3 \\
\hline & Partially viable & 1 \\
\hline & $<10 \%$ viable & 0 \\
\hline \multirow{4}{*}{ Subchondral bone } & Normal & 3 \\
\hline & Increased remodeling & 2 \\
\hline & Bone necrosis/granulation tissue & 1 \\
\hline & Detached/fractue/callus at base & 0 \\
\hline \multirow[t]{2}{*}{ Cartilage mineralization } & Normal & 3 \\
\hline & Abnormal/inappropriate location & 0 \\
\hline \multicolumn{2}{|l|}{ Average } & $(0-3)$ \\
\hline
\end{tabular}

Table 3: ICRS histological grading scale.

(SD). The changes in RV in relation to the time course were analyzed using the paired t-test. The significant differences in the remaining percentages of RV, modified 2D-MOCART scores, and ICRS scores were analyzed between the implanted sites and the controls, using Student's t-test (Excel, Microsoft). Differences with $\mathrm{p}<0.05$ were considered to be statistically significant.

\section{Results}

\section{CT images and radiolucent capacity of the defects}

A radiopaque area emerged from the boundary between the bone 
Citation: Murata D, Tokunaga S, Akieda S, Nakayama K, Setoyama K, et al. (2016) Osteochondral Regeneration of the Loading-bearing Site Using a Scaffold Free Three-dimensional Construct of Adipose Tissue-derived Mesenchymal Stem Cells in Pigs. J Tissue Sci Eng 7: 165. doi:10.4172/2157-7552.1000165

Page 4 of 8

and the implant in the defects and increased more steadily upward and inward on the implanted sites than the control sites (Figure 2a). The average RV (radiolucent volume) values in the both defects were significantly $(\mathrm{p}<0.02)$ decreased at the third $\left(0.10 \pm 0.04\right.$ and $0.12 \mathrm{~mm}^{3}$ $\pm 0.04 \mathrm{~mm}^{3}$ in the implanted and the control sites, respectively) and

A
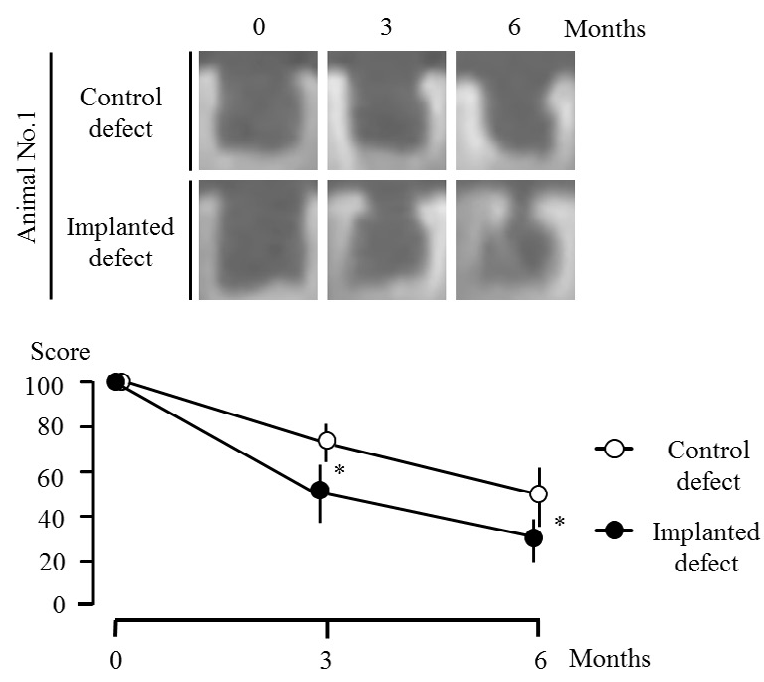

Figure 2: CT assessment of osteochondral defects. CT images show one cross-section of the multi-planar reconstruction images one, three, and six months after the surgery in animal No. 1 as a representative of all animals (a) Line graph shows the averages of RV (radiolucent volume) percentages at the third and sixth months against those at month zero in both defects (b). The averages at the third and sixth months were significantly (asterisks) decreased in the implanted defects, compared with the control defects. $\mathrm{P}<0.05$ was considered to be statistically significant. the sixth months $\left(0.06 \pm 0.02\right.$ and $0.08 \pm 0.03 \mathrm{~mm}^{3}$ in the implanted and the control sites, respectively) after the surgery, compared with those immediately after the surgery (at month zero, $0.20 \mathrm{~mm}^{3} \pm 0.04$ $\mathrm{mm}^{3}$ and $0.17 \mathrm{~mm}^{3} \pm 0.06 \mathrm{~mm}^{3}$ in the implanted and the control sites, respectively). The RVs percentages at the third and sixth months against those at month zero (that is, the remaining percentages of RV) were significantly decreased in the implanted defects $(52.5 \% \pm 16.5 \%$ and $30.2 \% \pm 10.4 \%$, respectively), compared with the control defects $(74.5 \%$ $\pm 8.0 \%$ and $50.5 \% \pm 13.6 \%$, respectively) (Figure $2 \mathrm{~b}$ ).

\section{MR images and modified MOCART scores of the defects}

MR images in the implanted sites of animal Nos. 1, 3, and 4 showed that the signal pattern at the surface of the articular cartilage was restored near to the normally-detected signal intensity of the surrounding sound cartilage, whereas the formation of new bone under the cartilaginous tissue was incomplete (Figure 3a). High signal intensity indicated that AT predominantly occupied the subchondral area in the implanted sites of animal Nos. 2 and 5. There were significant differences in the outcome measures such as defect fill $(\mathrm{p}<0.05)$, cartilage interface $(\mathrm{p}<0.05)$, and signal intensity $(\mathrm{p}<0.01)$ between the implanted and the control sites (Figure $3 \mathrm{~B})$. The scores of surface $(\mathrm{p}=0.06)$, cartilage structure, subchondral lamina and bone and adhesion $(\mathrm{p}=0.34)$ did not differ between both sites (Figure 3b). Accordingly, the total scores of the modified-MOCART system were significantly increased in the implanted sites $(36.0 \pm 17.8)$ compared with the controls $(15.0 \pm 0.0)$ (Figure 3b).

\section{Pathologic evaluation of the defects}

Gross pathology in the implanted sites of animal Nos. 1, 3, and 4 showed higher scores at the points of cartilage, including the coverage, the smooth surface, disappearing margin, and the color (Figure

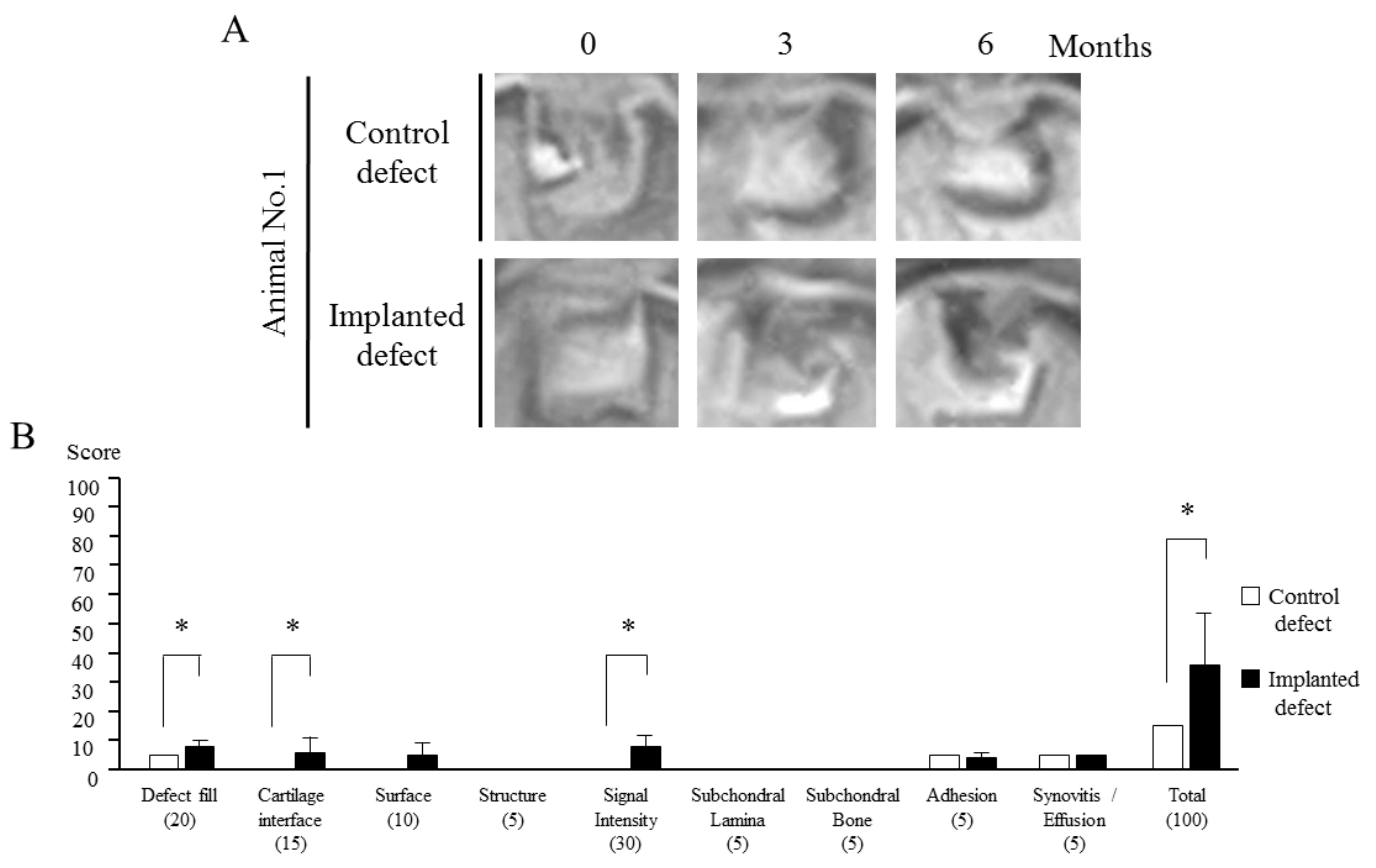

Figure 3: MR images of the osteochondral defects (A) and modified 2D-MOCART scores of osteochondral defects (B). MR images show one cross-section of the multiplanar reconstruction images one, three, and six months after surgery in animal No.1 as a representative of all of the animals (A). MR image in the implanted site of animal No. 1 showed that the articular cartilage was almost restored the normally-detected signal intensity of the surrounding sound cartilage, whereas the new bone formation under the cartilaginous tissue was incomplete. There were significant differences (asterisks) in the outcome measures such as defect fill, cartilage interface, signal intensity, and the total scores between the implanted and control sites $(B) . P<0.05$ was considered to be statistically significant. 
Citation: Murata D, Tokunaga S, Akieda S, Nakayama K, Setoyama K, et al. (2016) Osteochondral Regeneration of the Loading-bearing Site Using a Scaffold Free Three-dimensional Construct of Adipose Tissue-derived Mesenchymal Stem Cells in Pigs. J Tissue Sci Eng 7: 165. doi:10.4172/2157-7552.1000165

Page 5 of 8

4a). However, there were no significant differences in the outcome measures of ICRS gross scores such as coverage $(\mathrm{p}=0.13)$, neocartilage color ( $\mathrm{p}=0.13)$, defect margin ( $\mathrm{p}=0.37)$, and surface $(\mathrm{p}=0.09)$ between the implanted and the control sites (Figure $4 \mathrm{~b}$ ). Accordingly, the average gross scores did not significantly differ between the sites $(2.3 \pm 1.5$ and $1.0 \pm 0.7$ in the implanted and the control sites, respectively) (Figure 4b). Histopathology findings in animal Nos. 1, 3 , and 4 indicated the smooth coverage containing cartilage matrix positively stained by safranin $\mathrm{O}$ and immunohistochemistry and the subchondral bone formation in the implanted sites (Figure 5a and Figure $5 b$ ), while a deeply recessed surface and a lower rate of bone formation were observed in the control defects (Figure $5 \mathrm{a}$ and Figure $5 b)$. The ICRS histologic scores at the points except for subchondral bone were higher in the implanted sites than the control sites in animal Nos. 1, 3, and 4. Animal Nos. 2 and 5 showed that cartilagenous smooth coverage as well as the subchondral bone did not progress in both defects, therefore the scores at all points did not differ between the defects (Figure 5c). The scores of the histologic outcome measures such as surface, matrix, cell distribution, viability of the cell population, and cartilage mineralization (except for the subchondral bone $(\mathrm{p}=0.14))$ were significantly higher in the implanted sites than those in the control sites (Figure $5 c$ ). The average histological scores were also significantly higher in the implanted sites $(1.7 \pm 1.0)$ than in the control sites $(0.3 \pm 0.2)$ (Figure $5 c$ ).

\section{Discussion}

A radiopaque area emerged from the boundary between the implant and the bone defect, and RVs in both defects were significantly decreased three and six months after the surgery, compared with those immediately after the surgery. The results suggest that the subchondral bone formation naturally progressed in both osteochondral defects regardless of AT-MSCs implantation. However, the ratios (\%) of RVs at three and six months after surgery compared with those at month zero were significantly lower in the implanted sites than in the controls. This indicates that the increased radiopaque area after the surgery could be larger in the implanted sites than in the controls, and that the subchondral bone formation would be increased by the AT-MSCs implantation. The implantation of a scaffold-free 3D construct of ATMSCs could be a promising medical strategy for the regeneration of the subchondral bone lost in the clinical cases of OA. As presented in this study, the significant effects on bone formation were confirmed three months after the implantation using a quantitative analysis of $\mathrm{RV}$ in the CT images. On the other hand, given that percentages of $\mathrm{RV}$ in the $6^{\text {th }}$ month after the surgery were $30.2 \% \pm 10.4 \%$ and $50.5 \%$ $\pm 13.6 \%$ in the implanted and the control defects, respectively, the subchondral bone formation was not completed for six months in this study. We also performed a preliminary study to evaluate for one year on regeneration of the osteochondral defect in diameter of $6.8 \mathrm{~mm}$ and height of $10 \mathrm{~mm}$ (Supplemental Figure 1), and obtained the data showing lower percentages of RV persisting twelve months after AT-
A
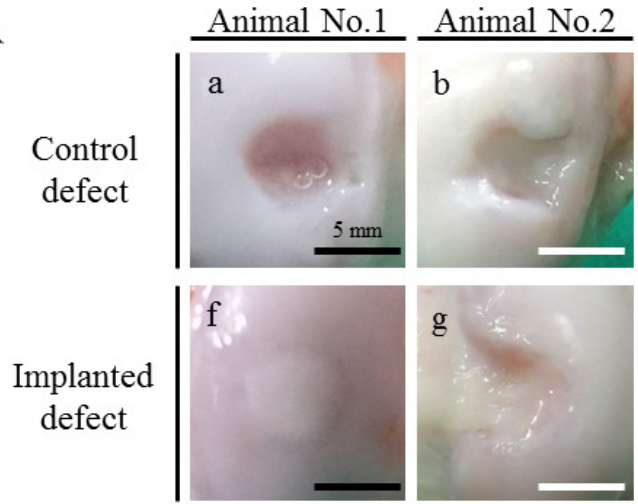
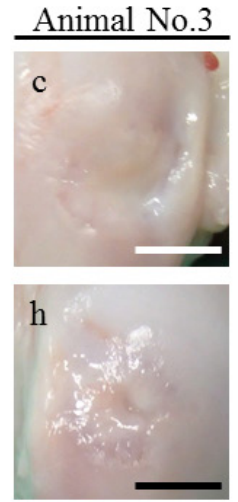
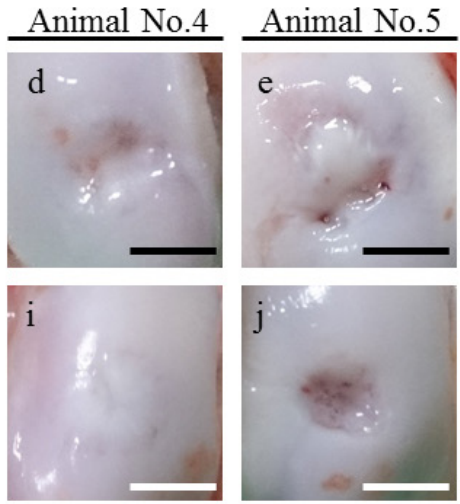

$\mathrm{B}$

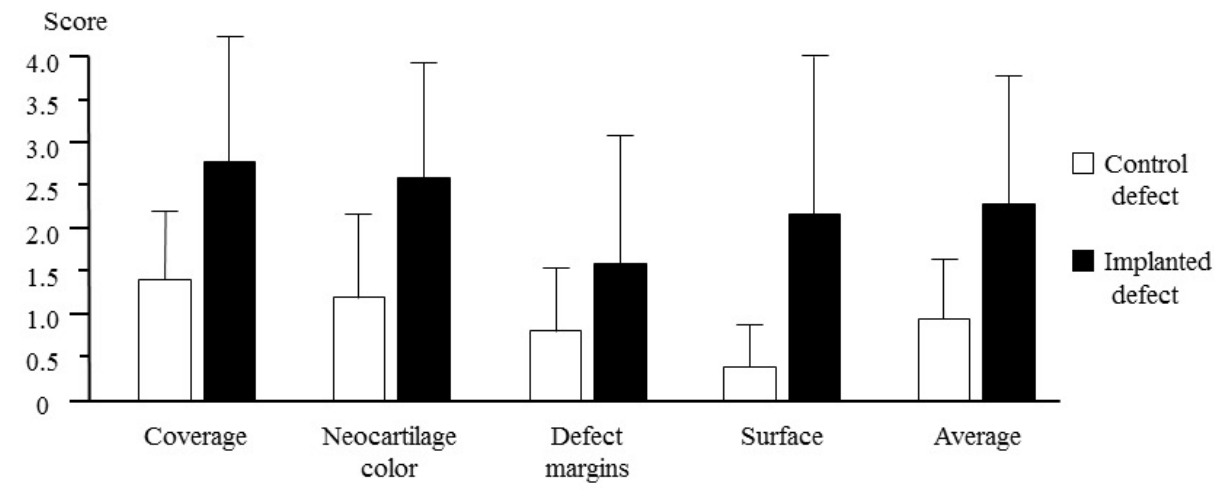

Figure 4: Macroscopic findings of the surface of the implanted and control sites (A) and macroscopic scores of the implanted and control sites with ICRS gross grading scale (B).

In animal Nos. 1, 3, and 4, the surfaces of the implanted defects were covered with the abundant cartilaginous white tissue (f, h, i), whereas the surfaces of the implanted defects were depressed, and the cartilaginous tissues was scarce in animal Nos. 2 and $5(\mathrm{~g}$, j). Furthermore, synovium adhered to the defect in animal No. 2 (g). On the other hand, the defects of the control sites were largely depressed in all animals (a, b, c, d, e). Gross pathology in the implanted sites of animal Nos. 1, 3, and 4 showed higher scores at the points of cartilage, including the coverage, the smooth surface, disappearing margin, and the color. However, there were no significant differences in the outcome measures of ICRS gross scores (B). 
Citation: Murata D, Tokunaga S, Akieda S, Nakayama K, Setoyama K, et al. (2016) Osteochondral Regeneration of the Loading-bearing Site Using a Scaffold Free Three-dimensional Construct of Adipose Tissue-derived Mesenchymal Stem Cells in Pigs. J Tissue Sci Eng 7: 165. doi:10.4172/2157-7552.1000165

A

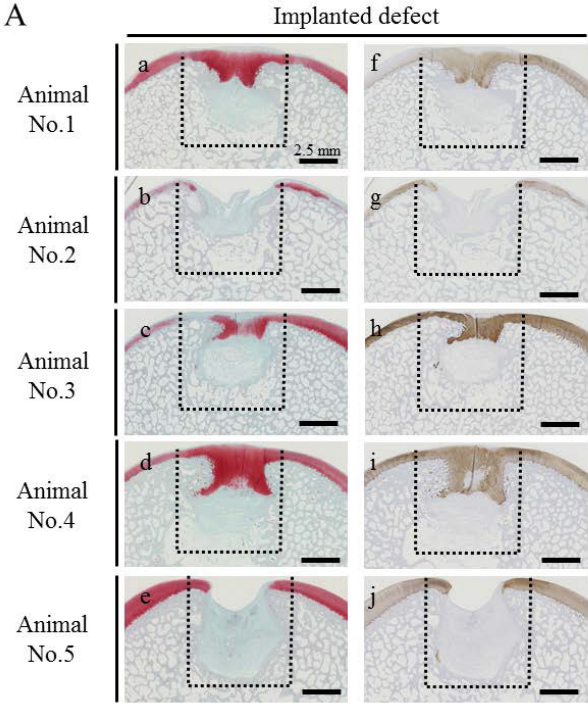

B

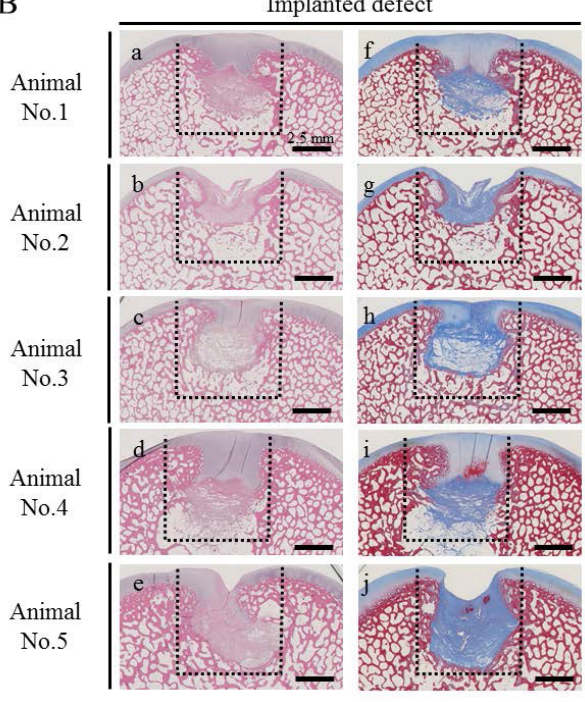

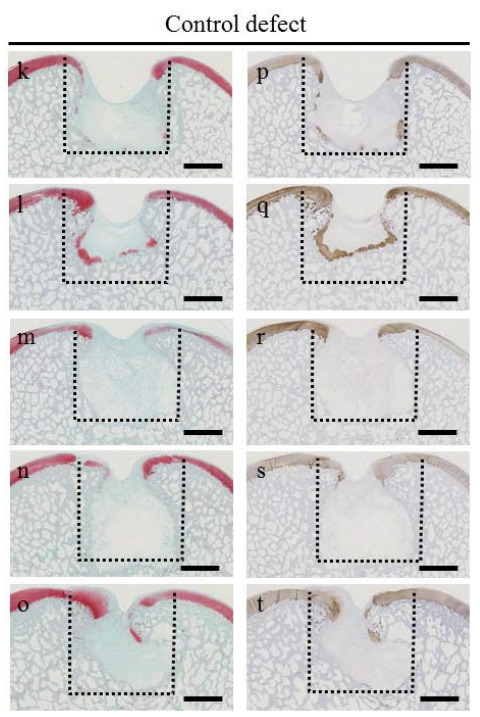

Control defect

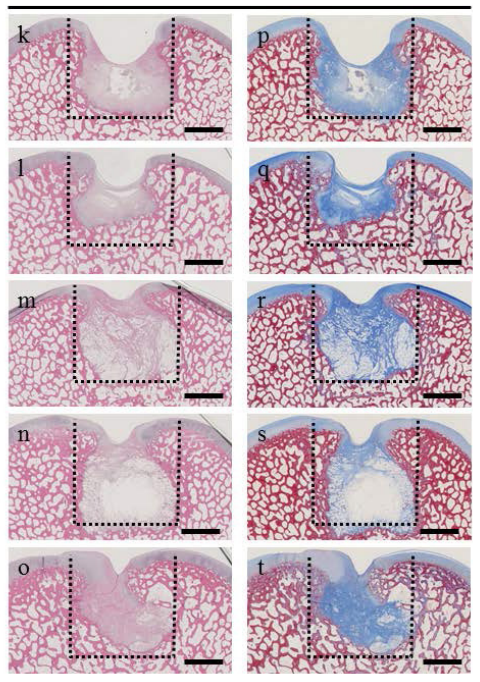

$\mathrm{C}$

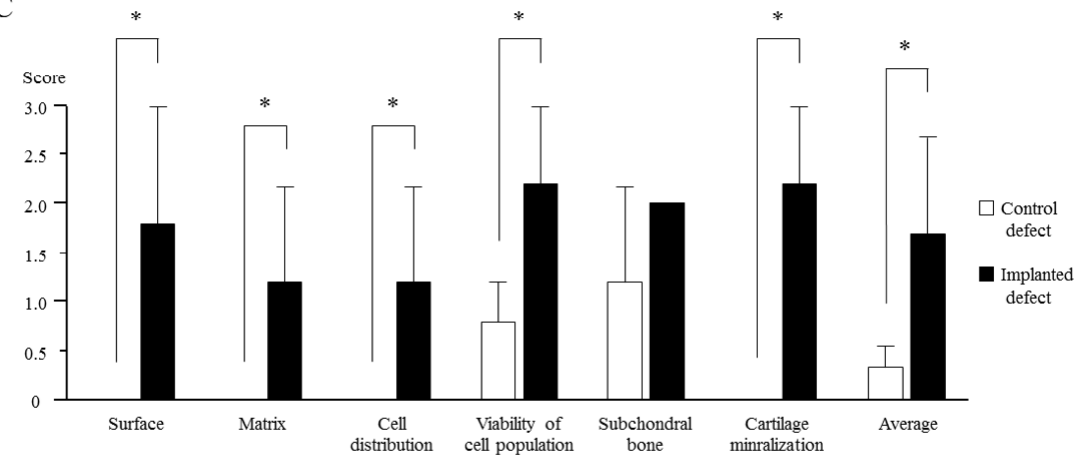

Figure 5: Histopathology of osteochondral defects using HE, Masson's trichrome, Safranin O, and immunohistochemical staining of type II collagen (A, B) and microscopic scores of the implanted and control sites with the ICRS histological grading scale (C). In animal Nos. 1, 3, and 4, smooth coverage containing cartilage matrix was positively stained with safranin $\mathrm{O}$ and immunohistochemistry (Aa, Ac, Ad, Af, Ah, Ai), and subchondral bone formation was observed on at the implanted sites $(\mathrm{Ba}, \mathrm{Bc}, \mathrm{Bd}, \mathrm{Bf}, \mathrm{Bh}, \mathrm{Bi})$, whereas the deeply recessed surface and the lower bone formation were left in the implanted sites in animal Nos. $2 \mathrm{and} 5$ ( $\mathrm{Ab}, \mathrm{Ae}, \mathrm{Ag}, \mathrm{Aj}$, $\mathrm{Bb}, \mathrm{Be}, \mathrm{Bg}, \mathrm{Bj}$ ). At the control site, the surface was irregular and fibrous tissue (which is not stained by safranin $\mathrm{O}$ ) covered the subchondral bone (Ak, Al, Am, An, Ao, $\mathrm{Ap}, \mathrm{Aq}, \mathrm{Ar}, \mathrm{As}, \mathrm{At}, \mathrm{Bk}, \mathrm{Bl}, \mathrm{Bm}, \mathrm{Bn}, \mathrm{Bo}, \mathrm{Bp}, \mathrm{Bq}, \mathrm{Br}, \mathrm{Bs}, \mathrm{Bt})$. The black dotted lines indicate the areas of osteochondral defects immediately after the surgery. The ICRS histologic scores at various points except for subchondral bone were higher in the implanted sites than in the control sites in animal Nos. 1, 3, and 4. Animal Nos. 2 and 5 showed that the cartilaginous smooth coverage as well as the subchondral bone did not progress in both defects; therefore the scores at all of the points did not differ between the defects. The scores of the histologic outcome measures such as surface, matrix, cell distribution, viability of cell population, and cartilage mineralization, and the average was significantly (asterisks) higher in the implanted sites than in the control sites (C). 
Citation: Murata D, Tokunaga S, Akieda S, Nakayama K, Setoyama K, et al. (2016) Osteochondral Regeneration of the Loading-bearing Site Using a Scaffold Free Three-dimensional Construct of Adipose Tissue-derived Mesenchymal Stem Cells in Pigs. J Tissue Sci Eng 7: 165. doi:10.4172/2157-7552.1000165

Page 7 of 8

MSCs implantation (7.0\% and $37.4 \%$ in the implanted and the control defects, respectively) (Supplemental Figure 2). Given these results, we speculate that the subchondral bone regeneration after the AT-MSC implantation progresses over one year based on the size of this defect (diameter of $6.8 \mathrm{~mm}$ and height of $10 \mathrm{~mm}$ ) (Supplemental Figure 3).

Proton density (PD) - MR images after six months after the surgery demonstrated that, in three of the animals (Nos. 1, 3, and 4), the double structure of the superficial and deep cartilage layers showing the high and the low signal layers, respectively, was nearly restored to that of the surrounding sound cartilage in the implanted sites, but not in the controls. The cartilage regeneration suggest by the MR imaging findings was correspondingly defined based on the higher gross scores of the coverage, the smooth surface, the disappearing margin, and the color in the implanted sites of these three animals. Similarly, in the other animals (Nos. 2 and 5), as their MR images lacked the signal pattern specific to articular cartilage, the cartilage scores were degraded in the gross pathology. Considering these results, MR imaging could be very helpful for speculating about cartilage regeneration by implanting a scaffold-free 3D construct of AT-MSCs. Recent studies have suggested that PD-MR imaging would be feasible for assessing injuries and matrix degradation in articular cartilage [15]. Some scaffolds, which can be detectable using MR imaging might conceivably mask the cartilage regeneration. Although MR imaging might not replace arthroscopy as the gold standard for cartilage evaluation, rapidly advancing MR imaging technology would be a powerful complementary tool. The significantly higher total scores with the modified- MOCART system in the implanted defect as presented here, suggest that MR imaging could become a non-invasive approach to the examination of cartilage regeneration using a scaffold-free 3D construct of AT-MSCs. The MR imaging also provided the insight into the osteogenesis or the adipogenesis occurring under the new cartilaginous tissues. The undesirable adipogenesis in the subchondral area after the implantation could be detectable using MR imaging, although we do not have a clear answer regarding whether MR imaging is more effective for speculating about subchondral bone formation compared with the CT imaging.

In animal Nos. 1, 3, and 4, gross pathology of the implanted sites showed higher scores at the points of cartilage, including the coverage, the smooth surface, disappearing margin, and the color than that of the control sites. However, in animal Nos. 2, and 5, there were little differences between the implanted and the control sites. As speculated the reason in the following section, no regeneration of either cartilage or bone in these two animals could result in the non-significant differences in macroscopic scores between them.

Histologically, in three animals (Nos. 1, 3, and 4), the smooth coverage containing the cartilage matrix was positively stained with safranin $\mathrm{O}$, and subchondral bone formation occurred in the implanted sites, whereas a deeply recessed surface and the lower levels of bone formation were observed in the control defects. The other animals (Nos. 2 and 5) showed that neither the cartilaginous smooth coverage nor the subchondral bone was formed in both defects. Consistent with the statistical analysis of the animal data, not only the individual histological scores such as surface, matrix, cell distribution, viability of cell population, and cartilage mineralization but also the average scores were higher in the implanted sites compared with control sites. The significant differences were conclusive evidence in support of our speculation that a scaffold-free 3D construct of AT-MSCs can possibly achieve osteochondral regeneration in the future. However, the reason why neither the cartilaginous coverage nor the subchondral bone was regenerated in the implanted defects of animal Nos. 2 and
5 warrant discussion. We speculated that the result could be due to defluxion of the AT-MSC constructs from the osteochondral defects in the early phase of post-operation. As mentioned in the preceding section, a 3D construct was accomplished by merging approximately 1,920 spheroids including $5 \times 10^{4}$ AT-MSCs individually. We suppose that the indissoluble merge between some spheroids might contribute to the fragility of the construct. Although the fragility has not yet been quantified, the vulnerable construct could be deformed during the surgery. As the constructs were implanted into the osteochondral defects in the femoral medial condyle, in this study, we cannot rule out the possibility of defluxion of the AT-MSC spheroids or constructs from the osteochondral defects in the early post-operative phase due to the movements of the joint. We should improve the quality of constructs as well as the surgical procedure of implantation in order to obtain more the creadibility of osteochondral regeneration by AT-MSCs.

Although further studies might be required, we conclude that implantation of a scaffold-free 3D construct of only AT-MSCs into an osteochondral defect will be able to regenerate the original structure of the cartilage and subchondral bone in load bearing site of large animal.

\section{Acknowledgements}

We express our gratitude to Dr. Yoshinao Hosaka, because of the arrangement for the experimental animals. We also thank Yudai Miyazaki and Manami Tsuji fo their valuable technical support to construct to prepare 3D constructs of AT-MSCs.

\section{Author's Contributions}

Daiki Murata cultured AT-MSCs, associated with the surgery to implant a 3D construct of AT-MSCs, and analyzed the diagnostic images and the pathological data. Satoshi Tokunaga evaluated the osteochondral defects by CT and MRI. Shizuka Akieda prepared the 3D constructs of AT-MSCs. Koichi Nakayama is an original proposer of the therapeutic strategy using a 3D construct of cells, and provided the suggestion to proceed this study. Kentaro Setoyama took care of the experimental animals. Makoto Fujiki is the operating surgeon at the time of implantation. Kazuhiro Misumi designed this study, supervised the project, and prepared the manuscript. All authors approved the final version of the manuscript.

\section{Funding Source}

This study was supported by Japan Society for the Promotion of Science (grant no. $15 \mathrm{H} 04600$ to Kazuhiro Misumi). The study sponsor had no involvement in this study design, collection, analysis, or interpretation of data; in the writing of the manuscript; or in the decision to submit the manuscript for publication.

\section{Disclosure of Interests}

Koichi Nakayama is a co-founder of Cyfuse Biomedical K.K. Shizuka Akieda is a full-time employee of the same company. The other authors have no commercial, proprietary, or financial interest in the products or companies described in this article.

\section{References}

1. Mankin HJ (1982) The response of articular cartilage to mechanical injury. J Bone Joint Surg Am 64: 460-466.

2. Ding C, Cicuttini F, Scott F, Cooley H, Boon C, et al. (2006) Natural history of knee cartilage defects and factors affecting change. Arch Intern Med 166 : 651-658.

3. Szerb I, Hangody L, Duska Z, Kaposi NP (2005) Mosaicplasty: long-term follow-up. Bull Hosp Jt Dis 63: 54-62.

4. Funayama A, Niki $Y$, Matsumoto $H$, Maeno S, Yatabe T, et al. (2008) Repair of full-thickness articular cartilage defects using injectable type II collagen gel embedded with cultured chondrocytes in a rabbit model. J Orthop Sci 13: 225232

5. Fujisato T, Sajiki T, Liu Q, Ikada Y (1996) Effect of basic fibroblast growth factor on cartilage regeneration in chondrocyte-seeded collagen sponge scaffold. Biomaterials 17: 155-162.

6. Diaz Romero J, Gaillard JP, Grogan SP, Nesic D, Trub T, et al. (2005) Immunophenotypic analysis of human articular chondrocytes: changes in surface markers associated with cell expansion in monolayer culture. J Cell Physiol 202: 731-742. 
Citation: Murata D, Tokunaga S, Akieda S, Nakayama K, Setoyama K, et al. (2016) Osteochondral Regeneration of the Loading-bearing Site Using a Scaffold Free Three-dimensional Construct of Adipose Tissue-derived Mesenchymal Stem Cells in Pigs. J Tissue Sci Eng 7: 165. doi:10.4172/2157-7552.1000165

Page 8 of 8

7. Tatebe M, Nakamura R, Kagami H, Okada K, Ueda M (2005) Differentiation of transplanted mesenchymal stem cells in a large osteochondral defect in rabbit. Cytotherapy 7: 520-530.

8. Pittenger MF, Mackay AM, Beck SC, Jaiswal RK, Douglas R, et al. (1999) Multilineage potential of adult human mesenchymal stem cells. Science 284 : 143-147.

9. Zuk PA, Zhu M, Ashjian P, De Ugarte DA, Huang JI, et al. (2002) Human adipose tissue is a source of multipotent stem cells. Mol Biol Cell 13: 4279-4295.

10. Nimura A, Muneta T, Koga H, Mochizuki T, Suzuki K, et al. (2008) Increased proliferation of human synovial mesenchymal stem cells with autologous human serum: Comparisons with bone marrow mesenchymal stem cells and with fetal bovine serum. Arthritis Rheum 58: 501-510.

11. Nakamura T, Sekiya I, Muneta T, Hatsushika D, Horie M, et al. (2013) Arthroscopic, histological and MRI analyses of cartilage repair after a minimally invasive method of transplantation of allogeneic synovial mesenchymal stromal cells into cartilage defects in pigs. Cytotherapy 14: 327-338.

12. Ishihara K, Nakayama K, Akieda S, Matsuda S, Iwamoto Y (2014) Simultaneous regeneration of full-thickness cartilage and subchondral bone defects in vivo using a three-dimensional scaffold-free autologous construct derived from highdensity bone marrow-derived mesenchymal stem cells. J Orthop Surg Res 9 : 98.

13. Murata D, Tokunaga S, Tamura T, Kawaguchi $H$, Miyoshi N, et al. (2015) A preliminary study of osteochondral regeneration using a scaffold-free threedimensional construct of porcine adipose tissue-derived mesenchymal stem cells. J Orthop Surg Res 10: 35.

4. Nakayama K (2013) In vitro biofabrication of tissues and organs in Biofabrication: Micro- and Nano-fabrication, Printing, Patterning and Assemblies, William Andrew, Oxford

15. Goebel L, Orth P, Müller A, Zurakowski D, Bücker A, et al. (2012) Experimenta scoring systems for macroscopic articular cartilage repair correlate with the MOCART score assessed by a high-field MRI at 9.4 T--comparative evaluation of five macroscopic scoring systems in a large animal cartilage defect model. Osteoarthritis Cartilage 20: 1046-1055.

16. Rehnitz C, Kupfer J, Streich NA, Burkholder I, Schmitt B, et al. (2014) Comparison of biochemical cartilage imaging techniques at $3 \mathrm{~T} \mathrm{MRI}$. Osteoarthritis Cartilage 22: 1732-1742. 